\title{
Enhanced magnetic second-harmonic generation from resonant metasurfaces
}

\author{
Sergey Kruk, ${ }^{\dagger}$ Martin Weismann, ${ }^{\ddagger}$ Anton Yu. Bykov, ${ }^{\S}$ Evgeniy A. Mamonov, ${ }^{\S}$ \\ Irina A. Kolmychek, ${ }^{\S}$ Tatiana Murzina, ${ }^{\S}$ Nicolae C. Panoiu, $\ddagger$ \\ Dragomir N. Neshev, ${ }^{\dagger}$ and Yuri S. Kivshar ${ }^{*} \dagger$
}

Nonlinear Physics Centre and Centre for Ultrahigh Bandwidth Devices for Optical Systems (CUDOS), Australian National University, Canberra ACT 0200, Australia, Department of Electronic and Electrical Engineering, University College London, London WC1E7JE, UK, Photon Design Ltd, 34 Leopold Street, Oxford OX4 1TW, UK, and Physics Department, Lomonosov Moscow State University, Moscow 119991, Russia

E-mail: Sergey.Kruk@anu.edu.au

\begin{abstract}
We study, both experimentally and theoretically, the second-order nonlinear response from resonant metasurfaces composed of metal-dielectric nanodisks. We demonstrate that by exciting the resonant optical modes of the composite nanoparticles we can achieve strong enhancement of the second-harmonic signal from the metasurface.

*To whom correspondence should be addressed

${ }^{\dagger}$ Nonlinear Physics Centre and Centre for Ultrahigh Bandwidth Devices for Optical Systems (CUDOS), Australian National University, Canberra ACT 0200, Australia

${ }_{\ddagger}^{\ddagger}$ Department of Electronic and Electrical Engineering, University College London, London WC1E7JE,

IPhoton Design Ltd, 34 Leopold Street, Oxford OX4 1TW, UK

${ }^{\S}$ Physics Department, Lomonosov Moscow State University, Moscow 119991, Russia
\end{abstract} UK 
By employing a multipole expansion method for the generated second-harmonic radiation, we show that the observed enhancement is due to the magnetic dipolar and electric quadrupolar second order nonlinear response of the metasurface.

KEYWORDS: Nonlinear optics, second-harmonic generation, plasmonic nanoparticles, optical magnetism, multipole decomposition

The nonlinear optical properties of nanostructures are known to differ substantially from those of bulk media because they are affected by strong confinement and local resonances. ${ }^{1-7}$ It is well established that the strong field enhancement through formation of 'hot spots' can dramatically boost nonlinear effects in metallic nanoparticles. ${ }^{8-10}$ Importantly, in the case of metamaterials, the nano-patterning leads not only to more efficient nonlinear interaction, but also to completely new nonlinear regimes due to magnetic optical response of the constituent 'meta-atoms'. While exciting applications of the linear magnetic response of metamaterials for both metallic ${ }^{11-15}$ and dielectric ${ }^{16-18}$ structures have been readily achieved at infra-red and even optical frequencies, the magnetic nature of nonlinear optical phenomena in metamaterials is still largely unexplored.

We focus our attention on the process of second-harmonic generation (SHG), which is an even-order nonlinear process that vanishes in centrosymmetric materials. ${ }^{19}$ For small nanoparticles, efficient SHG may be observed due to several factors, including local field enhancement, deviation of the particle shapes from a symmetric one, surface effects, etc. ${ }^{20} \mathrm{Im}-$ portantly, the resonances in plasmonic and dielectric nanoparticles, combined with a strong enhancement of the optical near-field, as well as the effective overlap of the interacting optical modes, allow for multi-fold enhancement of SHG. Optical SHG from regular plasmonic nanostructures was first studied for arrays of metal nanoparticles of noncentrosymmetric shape. ${ }^{21-23}$ It was recognized that the local field distribution depends drastically on the size, shape, and mutual orientation of the nanoparticles, thus providing a way for design of artifi- 
cial materials with required nonlinear optical properties. ${ }^{24-26}$ Such nonlinearity engineering, resembles the engineering of nonlinear materials by chemical composition, where molecules of different symmetries can be selected to enable the required nonlinear polarization. ${ }^{27,28}$ However, the top-down approach enables the ultimate design of any arbitrary nonlinear polarization.

Most of the research in this field was performed for various types of (asymmetric) plasmonic nanoparticles where the field localization effects are much more pronounced. Metasurfaces exhibiting both electric and magnetic resonances have also been studied, including split-ring resonator metasurfaces ${ }^{29,30}$ and fishnets. ${ }^{31}$ However, a certain controversy has remained about the nature of the observed harmonic generation from such resonant metasurfaces, namely if it arise from the electric or magnetic nonlinear polarization of the metasurfaces. ${ }^{32-34}$ Therefore, further studies about the exact origin, electric or magnetic, of the process of SHG from resonant metasurfaces is required.
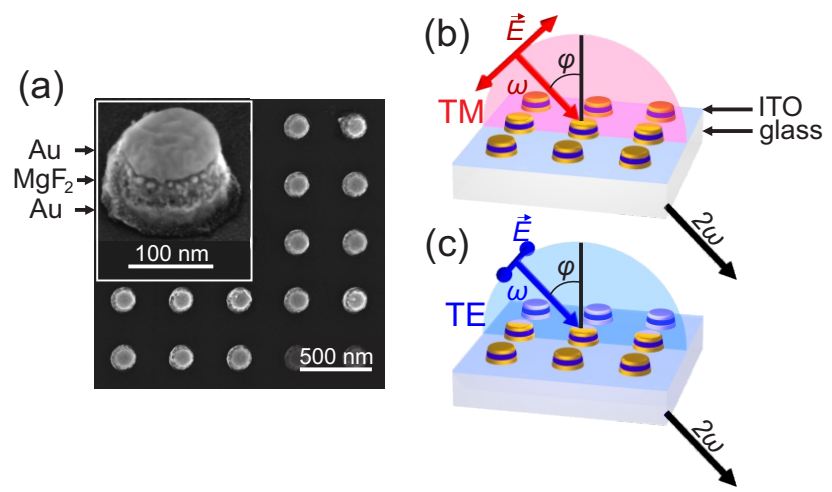

Figure 1: (a) SEM image of a fabricated metasurface. Inset shows a side view of a single three-layer meta-atom made of $\mathrm{Au} / \mathrm{MgF}_{2} / \mathrm{Au}$ layers. (b,c) Geometry of the SHG experiment for TM- and TE-polarized pump waves, respectively.

In this Letter, we analyze experimentally and theoretically the SHG from an optical metasurface of metal-dielectric-metal nanodisks in the vicinity of optically-induced electric and magnetic resonances. We demonstrate that resonant excitation of different modes of nanoparticles allows for strong enhancement of SHG signal near the optically-induced magnetic resonance. The related theoretical analysis further show that the radiated SHG intensity is a result of the electric quadrupolar and magnetic dipolar contributions of the 
nonlinear material polarization.

\section{Experimental results}

For our experiments, we employ metasurfaces formed by a regular array of metal-dielectricmetal disk-like nanoparticles (meta-atoms). We use two metal disks separated by a dielectric layer to obtain two distinct resonant modes of the meta-atom excited by incident light at two different wavelengths. ${ }^{35,36}$ The two resonances are associated with co- and counterpropagating electric currents excited in the parallel metal disks. In particular, the counterpropagating electric currents result in non-zero electric quadrupole and magnetic dipole linear response in the meta-atoms are therefore referred below as magnetic resonances of the metasurface.

The SEM image of the fabricated metasurface is shown in Fig. 1(a). The inset shows a side view of a single meta-atom. See Supplementary material for details on the fabrication procedure and sample dimensions. We study the second-order optical response of the metasurface for both TM and TE polarizations of the pump beam and different angles of incidence $\varphi$, as shown schematically in Figs. 1(b,c). The sample is illuminated from the top side (fundamental beam spot size of $\sim 50 \mu \mathrm{m}$ ) and the TM polarized second harmonic signal is collected from the substrate side.

First, we measure the linear transmission of the metasurface at normal incidence, as shown in Fig. 2(a, grey curve). The spectra are featured by two resonances centered at $640 \mathrm{~nm}$ and $790 \mathrm{~nm}$ wavelengths. The SHG spectroscopy is studied experimentally by employing a Titanium-Sapphire laser (100 fs pulses, $80 \mathrm{MHz}$ repetition rate, spectral range from $720 \mathrm{~nm}$ to $855 \mathrm{~nm}$ ) as a source of the fundamental radiation. The SHG radiation transmitted through the sample is spectrally selected by a set of color filters and a monochromator. Design parameters of the metasurface are chosen in such a way that the second resonance falls within the tunability range of the laser. The SHG signal is detected by spectral measurements and 

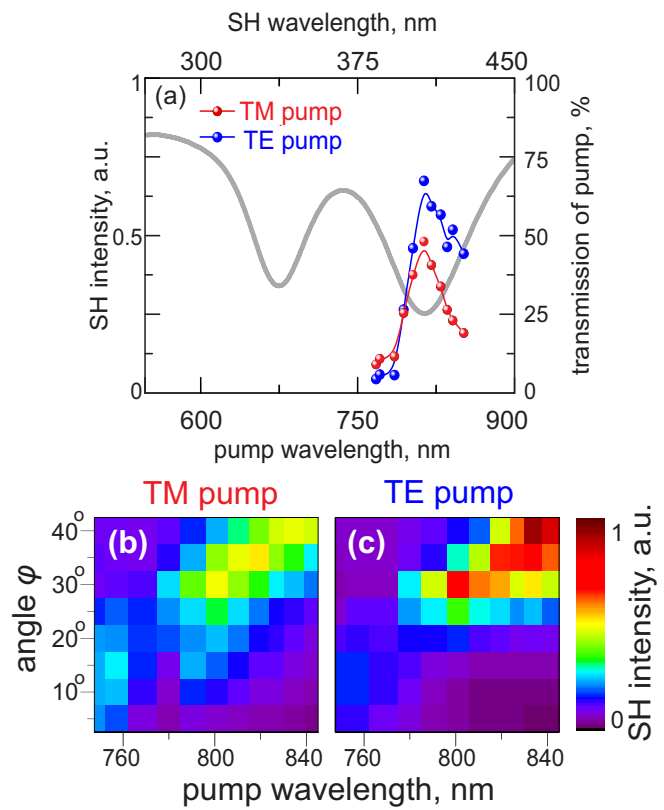

Figure 2: Experimental results. (a) Linear transmission of the metasurface (grey) at normal incidence and second harmonic spectra for TM- and TE-polarized pump waves (red and blue) measured for the incident angle of $30^{\circ}$. (b, c) Second-harmonic intensity vs. pump wavelength and angle of incidence for TM and TE polarizations.

measurements of a square dependence on the pump intensity.

Figure 2(a) shows the spectra of the SH intensity measured at the incidence angle $\varphi=30^{\circ}$ for two linear polarizations (red and blue, respectively). Both SH spectra show a pronounced efficiency enhancement near the resonance at $790 \mathrm{~nm}$ wavelengths. Figures 2(b,c) show the complete angular dependence of the SH spectra for the two polarizations. We note that at normal incidence the SHG efficiency nearly vanishes, as well as the SHG from the substrate remains below the noise level for all angles of incidence. The vanishing SHG at normal incidence is expected because the design of the metasurface elements is close to centrosymmetric, even with the presence of a substrate and a tilting shape of the metaatoms, and hence the SHG process is almost prohibited. In contrast, for oblique incidence surface dipoles contribute into the frequency conversion, and the SHG process becomes more efficient with an increase of the incident angle $\varphi$. In addition, we observe a red-shift of the SH spectral maxima with an increase of the incidence angle $\varphi$ for both TE and TM polarizations of the fundamental beam. 


\section{Theoretical insights}

In order to gain a deeper insight into our experimental results and the physical nature of the SHG process in resonant metasurfaces, we perform numerical calculations of the SH spectra and analyze its origin as a result of the emission of electric and magnetic multipoles. The normal-incidence linear transmission shown in Fig. 3(a, grey curve) is determined by using the generalized-source method (GSM). ${ }^{37}$ To validate these results, the transmission spectrum is also calculated by using CST Microwave Studio. The positions of the resonances in the calculated spectra are in a good agreement with the experimental data, corresponding to an electric resonance at approximately $640 \mathrm{~nm}$ and a magnetic one at $790 \mathrm{~nm}$. At the first resonance near $640 \mathrm{~nm}$, co-propagating currents are excited in the top and bottom metallic nanodisks of the meta-atom. At the second resonance, counter-propagating currents are excited in the two metallic nanodisks [see Fig. 3(b)]. However, the physical nature of these resonances is best revealed by applying the multipolar expansion method for the sources of the scattered field, namely the electric charges and currents of the meta-atom, in accord with the definitions,

$$
\begin{aligned}
\mathbf{p}_{\omega} & =\int \mathbf{P}_{\omega}(\mathbf{r}) d \mathbf{r} \\
\mathbf{m}_{\omega} & =-i \frac{\omega}{2} \int \mathbf{r} \times \mathbf{P}_{\omega}(\mathbf{r}) d \mathbf{r}, \\
Q_{\omega, j k} & =\int\left[3\left(r_{j} P_{\omega, k}+r_{k} P_{\omega, j}\right)-2 \delta_{j k} \mathbf{r} \cdot \mathbf{P}_{\omega}(\mathbf{r})\right] d \mathbf{r} .
\end{aligned}
$$

Here $\mathbf{P}_{\omega}(\mathbf{r})=\varepsilon_{0}\left[\varepsilon_{r}(\omega)-1\right] \mathbf{E}_{\omega}(\mathbf{r})$ is the polarization at the location point $\mathbf{r}$, with $\mathbf{E}_{\omega}(\mathbf{r})$ being the electric field and $\varepsilon_{r}$ the relative electric permittivity, and $\mathbf{p}_{\omega}, \mathbf{m}_{\omega}$, and $Q_{\omega, j k}$ are the electric dipole, magnetic dipole, and electric quadrupole of the nanodisk meta-atom, respectively. Importantly, Eqs. (1) include the contributions of both displacement and conductive currents if the frequency dispersion of $\varepsilon_{r}$ is taken into account. We notice that, since a contribution of the substrate into the far-field is shown to be negligible within the experimental 
accuracy, the integration in Eqs. (1) is restricted to the volume of the meta-atom.

We perform the multipolar expansion using Eqs. (1) that provides us with a quantitative analysis of the sources of the far-field at the pump. The results of this analysis are summarized in Figs. 4(a,b), and they suggest that the first resonance originates from the excitation of an electric dipole, whereas the second spectral peak corresponds to the excitation of a mixture of magnetic dipole and electric quadrupole moments resonantly excited together with the electric dipole. In addition, it can be seen that, for both types of the pump polarization, for wavelengths smaller than the wavelength of the magnetic resonance, the radiation emitted by the multipoles interfere constructively so that the total radiated power is higher than the power produced solely by the electric dipole. In the spectral region of the magnetic resonance and beyond, destructive interference is observed and the total radiated power becomes lower than that of the electric dipole alone.

The nonlinear optical response of the array of meta-atoms at the $\mathrm{SH}$ comprises several possible contributions, namely the surface (local) and bulk (nonlocal) SHG from the metallic nanodisks, as well as bulk (local) SHG from metallic disks and $\mathrm{MgF}_{2}$ dielectric layer. However, because of the centrosymmetric nature of the Au crystalline lattice and polycrystalline structure of $\mathrm{MgF}_{2}$, the last two contributions can be neglected. As a result, the sources of the $\mathrm{SH}$ field can be described by the following nonlinear polarizations, ${ }^{38}$

$$
\begin{aligned}
& \mathbf{P}_{\Omega}^{s}(\mathbf{r})=\epsilon_{0} \hat{\chi}_{s}^{(2)}: \mathbf{E}_{\omega}(\mathbf{r}) \mathbf{E}_{\omega}(\mathbf{r}) \delta\left(\mathbf{r}-\mathbf{r}_{s}\right), \\
& \mathbf{P}_{\Omega}^{b}(\mathbf{r})=\delta^{\prime}\left(\mathbf{E}_{\omega}(\mathbf{r}) \cdot \nabla\right) \mathbf{E}_{\omega}(\mathbf{r})+\beta \mathbf{E}_{\omega}(\mathbf{r})\left(\nabla \cdot \mathbf{E}_{\omega}(\mathbf{r})\right)+\gamma \nabla\left(\mathbf{E}_{\omega}(\mathbf{r}) \cdot \mathbf{E}_{\omega}(\mathbf{r})\right),
\end{aligned}
$$

where $\mathbf{P}_{\Omega}^{s}$ and $\mathbf{P}_{\Omega}^{b}$ describe the surface and bulk nonlinear polarization, respectively, and $\mathbf{r}_{s}$ defines the surface. The surface of metallic parts of the meta-atom possesses an isotropic mirror-symmetry plane perpendicular to the surface, so that the surface second-order susceptibility tensor, $\hat{\chi}_{s}^{(2)}$, has only three independent components, $\hat{\chi}_{s, \perp \perp \perp}^{(2)}, \hat{\chi}_{s, \perp\|\| \|}^{(2)}$, and $\hat{\chi}_{s,\|\| \perp}^{(2)}=$ 
$\hat{\chi}_{s,\|\perp\|}^{(2)}$, where the symbols $\perp$ (or $\left.\|\right)$ refer to the directions normal (or tangent) to the surface. For gold, the corresponding values are: ${ }^{39} \hat{\chi}_{s, \perp \perp \perp}^{(2)}=-(0.86+1.34 \mathrm{i}) \times 10^{-18} \mathrm{~m}^{2} \mathrm{~V}^{-1}, \hat{\chi}_{s,\|\| \perp}^{(2)}=-$ $(4.61+0.43 \mathrm{i}) \times 10^{-20} \mathrm{~m}^{2} \mathrm{~V}^{-1} \cdot \hat{\chi}_{s, \perp\|\| \mid}^{(2)}$ can be neglected as compared with other components, whereas the bulk susceptibility is $\gamma=7.13 \times 10^{-21} \mathrm{~m}^{2} \mathrm{~V}^{-1}$. Moreover, the dominant contribution to the bulk polarization comes from the third term in Eq. (2b), as in homogeneous media the second term vanishes and the ratio between the first and third terms is about $\nu / \omega$, where $\nu$ is the damping frequency (at optical frequencies, $\nu \ll \omega$ ); ${ }^{40}$ therefore, in our simulations we set $\delta^{\prime}=\beta=0$. Note that we did not include in Eq. (2b) a fourth term that accounts for the anisotropy of the medium because in our system the nonlinear bulk polarization is due entirely to the free electrons, namely it is generated in an isotropic medium.

To calculate the response at the SH frequency, we use a recently developed nonlinear extension to GSM. ${ }^{41}$ This nonlinear GSM is an efficient frequency domain method for solving the problems of light diffraction by periodic structures containing both linear and nonlinear optical media. It consists of three steps: $(i)$ Determine the electromagnetic field at the FF pump wavelength using the linear GSM; (ii) calculate nonlinear SH polarizations using Eqs. (2); and (iii) add this polarization to the linear polarization and use again the linear GSM to compute the electromagnetic fields at the SH frequency as well as the corresponding transmission and reflection coefficients. The SH spectra for two linear polarizations of pump determined by this approach, are shown in Fig. 3(a). Both SHG theoretical spectra reproduce well the experimental data, and they show pronounced maxima near the second (magnetic) resonance at around $790 \mathrm{~nm}$. Importantly, the theory predicts less efficient SHG near the first (electric) resonance at $640 \mathrm{~nm}$.

To understand the difference in the SHG efficiency observed at the two resonances, we employ a multipole expansion of the sources of the nonlinear field using a similar approach to that used at the FF. Specifically, we calculate numerically the distributions of nonlinear electric currents and charges induced in the meta-atoms by the optical pump and, subsequently, by using Eqs. (1) and (2), the nonlinear multipoles $\mathbf{p}_{\Omega}, \mathbf{m}_{\Omega}$, and $Q_{\Omega, i j}$ associated 
with these distributions. ${ }^{42,43}$ We emphasize that in these calculations we consider only the nonlinear source polarizations given in Eqs. (2), with the linear polarization of the medium at the SH frequency being excluded. Consequently, the resonant effects due to geometrical and material characteristics of the meta-atom on the radiative properties of these multipoles are not incorporated into our analysis, which implies that the multipoles are assumed to radiate as if they were in vacuum. By doing this, we want to separate the purely nonlinear properties of those multipoles from the linear properties in the spectral region of the secondharmonic generation. We follow this procedure to calculate the spectra of the first three terms of the multipolar expansion, namely the electric dipole, magnetic dipole, and electric quadrupole, induced in the meta-atom by a pump wave incident onto the metasurface at the angle $\varphi=30^{\circ}$.

Starting from the nonlinear optical response of a single meta-atom, we can now proceed to study the nonlinear properties of a metasurface created by a periodic square lattice of such meta-atoms as described by Eq. (11) of Supplementary material. ${ }^{?}$ The power spectra corresponding to the three types of multipoles, as well as the spectrum of the total emitted power are presented in Figs. 4(c) and 4(d) for both TM and TE polarized pump, respectively. While there are quantitative differences between these SHG spectra and those obtained using rigorous calculations based on the GSM [see Fig. 3(a)], both methods predict the same qualitative features of the SHG spectra, namely two spectral peaks corresponding to the electric and magnetic resonances, which are separated by a spectral region of weak SHG. More specifically, the radiated SHG intensity is dominated by the higher-order multipoles, electric quadrupole and magnetic dipole, with less than $3 \%$ contribution from the electric dipole.

Note that the spectral location of this weak SHG region, which can also be observed in the experimentally measured spectra [see Figs. 2(b) and 2(c)], is essentially independent of the angle of incidence of the FF beam. This observation confirms our theoretical predictions of a vanishing nonlinear dipole moment at this particular frequency. In addition, similarly 
to the linear optical response of the metasurface, these spectra illustrate that the nonlinear properties of the metasurface are determined by subtle interference effects among the radiation emitted by the three different types of multipoles, as observed in Figs. 4(c,d).

\section{Conclusions}

In conclusion, we have studied the second-harmonic generation from metasurfaces composed of metal-dielectric-metal nanodisks, which are known to support both electric and magnetic dipole resonances. We have experimentally observed that the intensity of the second-harmonic signal exhibits strong enhancement near the magnetic resonance of the metasurface, which agrees well with numerical calculations. We have shown that this strong enhancement of the second-harmonic intensity is a superposition of magnetic dipole and electric quadrupole modes induced by the pump wave at oblique incidence. We believe these

results will pave a way to establishing novel engineered nonlinear materials with enhanced harmonic efficiency, driven by optically-induced magnetic resonances.

\section{Acknowledgement}

The authors acknowledge useful comments from M. Kauranen and A. Zayats, as well as financial support from the Australian Research Council, the Russian Foundation for Basic Research (grant \#14-02-00446), and the UCL Impact Award graduate studentship funded by UCL and Photon Design Ltd.

\section{References}

(1) Kreibig, U.; Vollmer, M. Optical Properties of Metal Clusters; Springer-Verlag, Berlin, 1995.

(2) Barnes, W. L.; Dereux, A.; Ebbesen, T. W. Nature 2003, 424, 824. 
(3) Zayats, A. V.; Smolyaninov, I. I.; Maradudin, A. A. Phys. Reports 2005, 408, 131.

(4) Maier, S. A. Plasmonics: Fundamentals and Applications; Springer, New York, 2007.

(5) Panoiu, N. C.; Osgood, R. M. Nano Lett. 2004, 4, 2427-2430.

(6) Suh, J. Y.; Odom, T. W. Nano Today 2013, 8, $469-479$.

(7) Shcherbakov, M. R.; Neshev, D. N.; Hopkins, B.; Shorokhov, A. S.; Staude, I.; MelikGaykazyan, E. V.; Decker, M.; Ezhov, A. A.; Miroshnichenko, A. E.; Brener, I.; Fedyanin, A. A.; Kivshar, Y. S. Nano Lett. 2014, 14, 6488-6492.

(8) van Nieuwstadt, J. A. H.; Sandtke, M.; Harmsen, R. H.; Segerink, F. B.; Prangsma, J. C.; Enoch, S.; Kuipers, L. Phys. Rev. Lett. 2006, 97, 146102.

(9) Fan, W.; Zhang, S.; Panoiu, N.-C.; Abdenour, A.; Krishna, S.; Osgood, R. M.; Malloy, K. J.; Brueck, S. R. J. Nano Lett. 2006, 6, 1027.

(10) Kauranen, M.; Zayats, A. V. Nat. Photon. 2012, 6, 737-748.

(11) Pendry, J. B.; Holden, A. J.; Robbins, D. J.; Stewart, W. J. IEEE Trans. Microwave Theory Tech. 1999, 47, 2075.

(12) Linden, S.; Enkrich, C.; Wegener, M.; Zhou, J. F.; Koschny, T.; Soukoulis, C. M. Science 2004, 306, 1351-1353.

(13) Yen, T. J.; Padilla, W. J.; Fang, N.; Vier, D. C.; Smith, D. R.; Pendry, J. B.; Basov, D. N.; Zhang, X. Science 2004, 303, 1494-1496.

(14) Zhang, S.; Fan, W. J.; Minhas, B. K.; Frauenglass, A.; Malloy, K. J.; Brueck, S. R. J. Phys. Rev. Lett. 2005, 94, 037402.

(15) Butet, J.; Thyagarajan, K.; Martin, O. J. F. Nano Lett. 2013, 13, 1787-1792. 
(16) Etxarri, G. A.; Medina, G. R.; Perez, F. L. S.; Lopez, C.; Chantada, L.; Scheffold, F.; Aizpurua, J.; Vesperinas, N. M.; Saenz, J. J. Opt. Express 2011, 19, 4815-4826.

(17) Kuznetsov, A. I.; Miroshnichenko, A. E.; Fu, Y. H.; Zhang, J.; Lukyanchuk, B. Sci. Rep. 2012, 2, 492.

(18) Liu, S.; Sinclair, M. B.; Mahony, T. S.; Jun, Y. C.; Campione, S.; Ginn, J.; Bender, D. A.; Wendt, J. R.; Ihlefeld, J. F.; Clem, P. G.; Wright, J. B.; Brener, I. Optica $2014,1,250-256$.

(19) Burns, W. K.; Bloembergen, N. Phys. Rev. B 1971, 4, 3437-3450.

(20) Aktsipetrov, O.; Elyutin, P.; Nikulin, A.; Ostrovskaya, E. Phys. Rev. B 1995, 51, 17591-17599.

(21) Lamprecht, B.; Leitner, A.; Aussenegg, F. Appl. Phys. B 1999, 68, 419-423.

(22) Tuovinen, H.; Kauranen, M.; Jefimovs, K.; Vahimaa, P.; Vallius, T.; Turunen, J.; Tkachenko, N. V.; Lemmetyinen, H. J. Nonlin. Opt. Phys. E Materials 2002, 11, 421432.

(23) Canfield, B. K.; Kujala, S.; Jefimovs, K.; Turunen, J.; Kauranen, M. Opt. Express 2004, 12, 5418-5423.

(24) Kujala, S.; Canfield, B.; Kauranen, M.; Svirko, Y.; Turunen, J. Phys. Rev. Lett. 2007, 98, 167403.

(25) Czaplicki, R.; Husu, H.; Siikanen, R.; Makitalo, J.; Kauranen, M.; Laukkanen, J.; Lehtolahti, J.; Kuittinen, M. Phys. Rev. Lett. 2013, 110, 093902.

(26) Valev, V. K. et al. Adv. Mater. 2014, 26, 4074-4081.

(27) Zyss, J. Molecular nonlinear optics: materials, physics, and devices; Academic press Boston, 1994; Vol. 260. 
(28) Sioncke, S.; Verbiest, T.; Persoons, A. Materials Science and Engineering: R: Reports 2003, 42, $115-155$.

(29) Klein, M. W.; Enkrich, C.; Wegener, M.; Linden, S. Science 2006, 313, 502-504.

(30) Klein, M. W.; Wegener, M.; Feth, N.; Linden, S. Opt. Express 2007, 15, 5238-5247.

(31) Kim, E.; Wang, F.; Wu, W.; Yu, Z.; Shen, Y. Phys. Rev. B 2008, 78, 113102.

(32) Ciracì, C.; Poutrina, E.; Scalora, M.; Smith, D. R. Phys. Rev. B 2012, 85, 201403.

(33) Poutrina, E.; Huang, D.; Urzhumov, Y.; Smith, D. R. Opt. Express 2011, 19, 83128319.

(34) Rose, A.; Huang, D.; Smith, D. R. Phys. Rev. Lett. 2013, 110, 063901.

(35) Dolling, G.; Enkrich, C.; Wegener, M.; Zhou, J. F.; Soukoulis, C. M.; Linden, S. Opt. Lett. 2005, 30, 3198-3200.

(36) Shalaev, V. M.; Cai, W.; Chettiar, U. K.; Yuan, H.-K.; Sarychev, A. K.; Drachev, V. P.; Kildishev, A. V. Opt. Lett. 2005, 30, 3356-3358.

(37) Shcherbakov, A. A.; Tishchenko, A. V. J. Quant. Spectrosc. Radiat. Transfer 2012, $113,158-171$.

(38) Heinz, T. F. In Nonlinear Surface Electromagnetic Phenomena; Ponath, H. E., Stegeman, G. I., Eds.; Elsevier, Amsterdam, 1991; Chapter 5, pp 353-416.

(39) Krause, D.; Teplin, C. W.; Rogers, C. T. J. Appl. Phys. 2004, 96, 3626-3634.

(40) Wang, F. X.; Rodriguez, F. J.; Albers, W. M.; Ahorinta, R.; Sipe, J. E.; Kauranen, M. Phys. Rev. B 2009, 80, 233402.

(41) Weismann, M.; Gallagher, D. F. G.; Panoiu, N. C. J. Opt. Soc. Am. B submitted,

(42) Dadap, J. I.; Shan, J.; Heinz, T. F. J. Opt. Soc. Am. B 2004, 21, 1328. 
(43) Cao, L.; Panoiu, N. C.; Bhat, R. D. R.; Osgood, R. M. Phys. Rev. B 2009, 79, 235416. 


\section{Graphical TOC Entry}

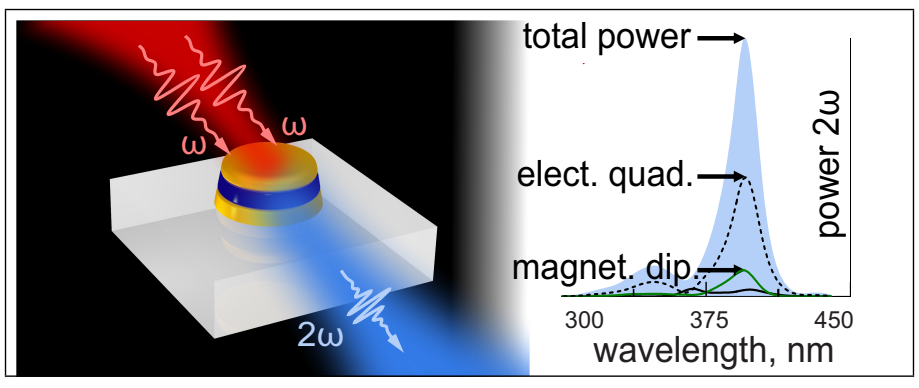




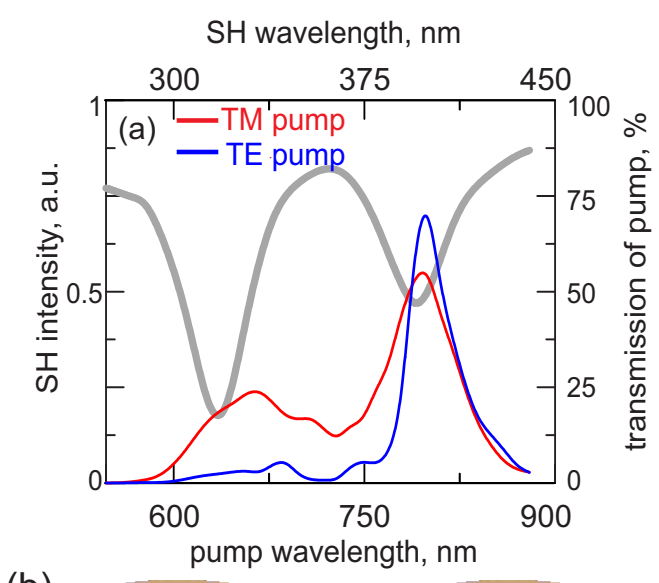

(b)

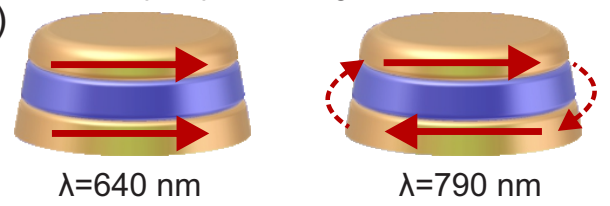

Figure 3: (a) Numerical transmission spectrum of the metasurface at normal incidence (grey) and SH spectra for TM- and TE-polarized pumps (red and blue, respectively) calculated for the incidence angle $\phi=30^{\circ}$. (b) Schematic of the electric currents distribution in a single meta-atom at the first and second resonant wavelengths. Dashed lines visualize the displacement current.

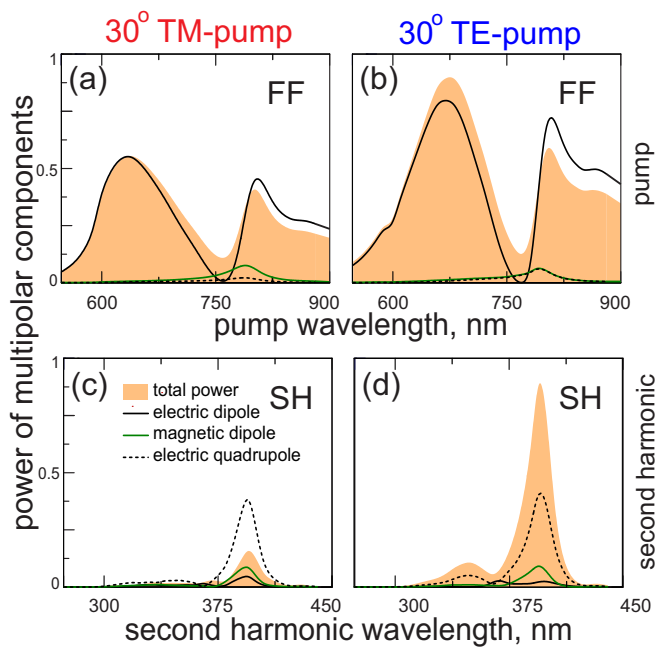

Figure 4: Numerically calculated cross sections of the electric dipole (black, solid), electric quadrupole (black, dashed), and magnetic dipole (green) at (a,b) fundamental wavelengths and $(\mathrm{c}, \mathrm{d})$ second-harmonic wavelengths, for two polarisations of the pump at the oblique incidence. 